\title{
Analysis of $g_{2}$ for the cold collision frequency shift in the hydrogen condensate experiments
}

\author{
C.W. Gardiner and A.S. Bradley \\ School of Chemical and Physical Sciences, Victoria University, Wellington, New Zealand
}

\begin{abstract}
We compute an approximate set of longitudinal quasiparticle modes for a hydrogen condensate as produced in the MIT experiments. An expansion in quasiparticles using a simple one-dimensional Bogoliubov picture shows however that at the high temperatures $(44 \mathrm{~K})$ and in the very shallow trap employed $(! z=2 \quad 10: 2 \mathrm{H} \mathrm{z})$ the contribution to the density from the quasiparticles is about $20 \%$ of that from the condensate mode, leading to an effective $g_{2}(x ; x)$ which varies between 1 and 3 depending on the position in the condensate.
\end{abstract}

\section{Introduction}

The analysis of the experimental data on a spin-polarized atomic hydrogen Bose-Einstein condensate as given in [6, 3, 5, 2], relates the density of the condensate or of the noncondensed gas to the frequency for the two-photon $1 \mathrm{~s}-2 \mathrm{~s}$ transition by the cold collision line-shift formula

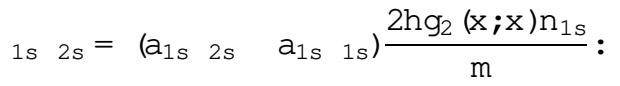

In this formula $a_{1 s} 1_{s}$ and $a_{1 s} \quad 2 s$ are respectively the scattering lengths for collisions between the two atoms in the $1 \mathrm{~s}$ state or of one atom in each state, $\mathrm{n}_{1 \mathrm{~s}}$ is the density of the $1 \mathrm{~s}$ atoms, and

$$
g_{2}\left(x ; x^{0}\right)=\frac{h^{y}(x){ }^{y}\left(x^{0}\right)\left(x^{0}\right)(x) i}{n_{1 s}(x) n_{1 s}\left(x^{0}\right)}:
$$

For a non-condensed thermal gas $g_{2}(x ; x)=2$, while for a pure condensate $g_{2}(x ; x)=1$.

The derivation of the formula (11) is given in [5], though it is acknowledged there that this derivation is only valid for special cases; that is for a spatially homogeneous thermal non-condensed gas, and for a pure condensate. Intermediate situations, such as those which appear to pertain in practice have not been treated, and indeed are very difficult to analyze.

In the experiments with the choice of $g_{2}(x ; x)=1$, frequency shifts measured in the Bose-Einstein condensate experiments would correspond to densities too high to be credible, and the data were analyzed using $g_{2}(x ; x)=2$. This was justified by the manifest high temperature of the system, and the conjecture that condensation occurred into a multiplicity of states.

This paper is the first of two on this subject. Here we shall concern ourselves specifically with the computation of $g_{2}$ for the case of hydrogen condensate with a significant thermal component, in order to demonstrate what would be predicted by its use if the formula (11) is assumed to be valid in that case. In our second paper [ $₫$ ] we shall show that the application of 
(1) to a condensate with a thermal component is not justified, and we shall give a full dynamic formulation of the actual processes involved.

The evaluation of $g_{2}$ is relevant to other phenomena, such as two-body loss processes, so its computation is still of value, even if it is not directly applicable to the analysis of the cold collision frequency shift in these experiments. Furthermore, in order to do the computations of our second paper, we shall need the eigenfunctions computed here. We will use a simple Bogoliubov model, though it will become apparent that there should still be significant corrections to this at the temperatures and densities found in practice. A more detailed study is left for a later paper.

One might expect that in general $g_{2}(x ; x)$ lies between 1 and 2 , but in fact the only fundamental limit is that $0 \quad g_{2}(x ; x) \quad 1$. However, in the case of Gaussian statistics, we get a stronger limit. Thus in the case of a fully thermalized gas, we can split the correlation function up using Gaussian factorization

$$
h^{y}(x)^{y}\left(x^{0}\right) \quad\left(x^{0}\right) \quad \begin{aligned}
(x) i= & h^{y}(x) \quad(x) \text { ih }{ }^{y}\left(x^{0}\right) \quad\left(x^{0}\right) i \\
& +h^{y}(x)\left(x^{0}\right) \text { ih }{ }^{y}\left(x^{0}\right) \quad(x) i \\
& +h^{y}(x){ }^{y}\left(x^{0}\right) \text { ih }\left(x^{0}\right) \quad(x) i
\end{aligned}
$$

The third term in the case of a thermal gas is zero since there is no anomalous average

$$
h^{y}(x){ }^{y}\left(x^{0}\right) i=0 \text {; }
$$

and setting $\mathrm{x}=\mathrm{x}^{0}$ gives

$$
g_{2}(x ; x)=\frac{h^{y}(x){ }^{y}(x) \quad(x) \quad(x) \text { i }}{h^{y}(x) \quad(x) \text { ih }^{y}(x) \quad(x) i} ! 2:
$$

Thus $g_{2}(x ; x)=2$ is a consequence of the Gaussian nature of the fluctuations and of the absence of an anomalous average. The anomalous average does have an upper bound of approximately

$$
\text { h }(x) \quad\left(x^{0}\right) i j<h^{y}(x) \quad(x) i
$$

which gives the bound

$$
g_{2}(x ; x)<3 \text { : }
$$

We will find in our calculation that in fact $g_{2}(x ; x)>2$ can occur in some regions of the Hydrogen vapor-condensate system for the experimentally realized parameters.

\section{Second-quantized Hamiltonian}

For the case under consideration the second-quantized Hamiltonian can be written in the form

$$
\mathrm{H}=\mathrm{d}^{3} \mathrm{x}^{\mathrm{n}} \mathrm{y}(\mathrm{T}+\mathrm{V}(\mathrm{x}))+\frac{\mathrm{u}}{2} \mathrm{y}^{\mathrm{O}} \mathrm{O}:
$$

i) The kinetic energy operator is $\mathrm{T}=\mathrm{h}^{2} \mathrm{r}^{2}=2 \mathrm{~m}$

ii) The interaction coefficient is given in terms of the scattering length by

$$
\mathrm{u}=4 \quad \mathrm{a}_{1 \mathrm{~s}} \quad 1 \mathrm{~s} \mathrm{~h}^{2}=\mathrm{m}
$$

Experimentally

$$
\begin{aligned}
& a_{1 \mathrm{~s}} 1 \mathrm{~s}=0: 0648 \mathrm{~nm} \\
& a_{1 \mathrm{~s}} 2 \mathrm{~s}=1: 4 \quad 0: 3 \mathrm{~nm}:
\end{aligned}
$$




\section{Evaluation of $g_{2}(x ; x)$ for a warm condensate}

The hydrogen condensate at MIT is formed at $44 \mathrm{~K}$, and the radial and axial trap frequencies are

$$
\begin{array}{ll}
!_{\mathrm{r}}=2 & 3: 9 \mathrm{kH} \mathrm{z} \\
!_{\mathrm{z}}=2 & 10: 2 \mathrm{H} \mathrm{z}:
\end{array}
$$

The ratio of these $!_{r}=!_{z}=382$ gives the trap aspect ratio $l_{z}=l_{x}$, which at nearly 400:1 gives an almost one-dimensional condensate.

As well as this, the ratios of the energies of the transverse and longitudinal quanta to the temperature are

$$
\begin{array}{ll}
\mathrm{kT}=\mathrm{h} !_{\mathrm{r}} & 1=250 \\
\mathrm{kT}=\mathrm{h} !_{\mathrm{z}} & 1=10^{5}:
\end{array}
$$

We can therefore expect that the average number of quasiparticles in the longitudinal mode is of the order of $10^{5}$ for the lower levels. This does not mean that the condensate is very different in size and shape, but its coherence may be affected by these large excitations. For example, the longitudinal Kohn mode corresponds to center of mass oscillations of the whole condensate, of mass $10^{9} \mathrm{~m}$, and using statistical mechanics, this means that

$$
\mathrm{P} \overline{\mathrm{hz}} \mathrm{z}^{2} \mathrm{i} \quad 3 \quad 10{ }^{4} \mathrm{~mm} \quad 6 \quad 10{ }^{6} \mathrm{l}_{\mathrm{z}} \text {; }
$$

which is a completely unobservable deviation.

\subsection{Approximate evaluation of mode functions}

In order to get an approximate idea of the thermal fluctuations we will compute the wavefunctions of the lower lying excitations using a hydrodynamic approximation based on the work of Zaremba [10]. This method gives an approximate form for the full three dimensional wavefunction in the case that the radial modes are not excited; thus it is possible do a calculation by means of a one dimensional wave equation, and using the Thomas-Fermi approximation for the condensate wavefunction this give eigenfunctions analytically in terms of Jacobi polynomials.

3.1.1. Hydrodynamic equations The system can be characterized by a density $\quad(x ; t)$ and a phase $(x ; t)$, and the hydrodynamic equations are written in terms of the linearized density fluctuation $(x ; t)=(x ; t) \quad 0(x)$ as

$$
\begin{aligned}
-(x ; t) & =\frac{h}{m} r \quad[0(x) r \quad(x ; t)] \\
-(x ; t) & =\frac{u}{h} \quad(x ; t):
\end{aligned}
$$

Zaremba's analysis considers only the situation in which there is a cylindrically symmetric condensate with no z-dependence, and he shows that in this case the speed of sound along the $z$ direction is half that expected in a homogeneous condensate with density equal to the peak density of the cylindrical condensate. We will generalize his result to the situation in

which the trap is harmonic in all directions, but is very weak along the $z$-direction. Following Zaremba's lead, we look for the equation of motion for a perturbation with the property

$$
\begin{aligned}
(x ; t) & =n(z) \text { when } o(x) \in 0 ; \\
& =0 \quad \text { when } 0(x)=0:
\end{aligned}
$$


Here it is assumed that the Thomas-Fermi form

$$
o(x)=\frac{2 \quad m !{ }_{r}^{2} r^{2} m !{ }_{z}^{2} z^{2}}{2 u} ;
$$

is used, so that (19) means that $\mathrm{n}(\mathrm{z})$ is only non-zero where there is a non-vanishing condensate density, i.e., when

$$
r<R(z) \quad \frac{S \frac{m ! z_{z}^{2} z^{2}}{m ! r}}{\frac{2}{r}}
$$

When we substitute into the wave equation for that arises from combining [17) and $[1$ ), and then integrate over $2 \mathrm{rdr}$, the resulting equation can be written in terms of the scaled length

$$
z=h \frac{s}{\frac{2}{m !_{z}^{2}}} h
$$

as

$$
\frac{a^{2} n}{a t^{2}}=\frac{!_{z}^{2}}{4} \quad\left(1 \quad h^{2}\right) \frac{a^{2} n}{a h^{2}} \quad 4 h \frac{a n}{\varrho h} \quad:
$$

The operator on the right hand side is that of the Jacobi polynomials $P_{n}^{(1 ; 1)}(h)$, leading to the eigenvalue spectrum

$$
!_{\mathrm{n}}=\frac{!_{\mathrm{z}} \mathrm{p}}{2} \overline{\mathrm{n}(\mathrm{n}+3)} \text { : }
$$

The more exact three dimensional analysis of Fliesser et al. [1] gives, in the case of an extended cigar-shaped condensate, the same formula.

Note that these Jacobi polynomials are orthogonal in the sense that

$$
\mathrm{Z}_{1} \mathrm{dh}\left(1 \mathrm{~h}^{2}\right) \mathrm{P}_{\mathrm{n}}^{(1 ; 1)}(\mathrm{h}) \mathrm{P}_{\mathrm{m}}^{(1 ; 1)}(\mathrm{h})=\frac{8(\mathrm{n}+1) \mathrm{n} ; \mathrm{m}}{(2 \mathrm{n}+3)(\mathrm{n}+2)} ;
$$

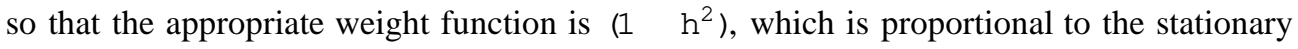
condensate density.

3.1.2. Three dimensional interpretation of the wavefunctions As in Zaremba's analysis, these eigenfunctions are constant in the radial direction to the edge of the Thomas-Fermi condensate (21), where they abruptly drop to zero. This means that the excitations are all essentially sound waves travelling along the length of the condensate.

3.1.3. Quasiparticle wavefunctions We can thus write

$$
\begin{aligned}
& \mathrm{n}(\mathrm{z} ; \mathrm{t})={ }^{\mathrm{X}} \mathrm{A}_{\mathrm{n}} \cos \left(!_{\mathrm{n}} \mathrm{t}\right) \mathrm{P}_{\mathrm{n}}^{(1 ; 1)}(\mathrm{h}) \\
& (\mathrm{z} ; \mathrm{t})={ }^{\mathrm{n}} \frac{\mathrm{uA_{n }}}{\mathrm{h} !_{\mathrm{n}}} \sin \left(!_{\mathrm{n}} \mathrm{t}\right) \mathrm{P}_{\mathrm{n}}^{(1 ; 1)}(\mathrm{h}):
\end{aligned}
$$

The resulting condensate wavefunction (normalized to the total number of particles, not to 1) is given by

$$
(x ; t)=p \frac{p(x)+(x ; t)}{\exp (i \quad(z ; t))}
$$


Analysis of $\mathrm{g}_{2}$ in hydrogen...

$$
\begin{aligned}
& \mathrm{p} \overline{o(\mathrm{x})}+\frac{\mathrm{p}}{2} \frac{(\mathrm{x} ; \mathrm{t})}{\mathrm{o(x)}}+i \overline{\mathrm{p}} \overline{o(\mathrm{x})} \quad(\mathrm{z} ; \mathrm{t}) \\
& =p \overline{o(x)} \\
& +{ }_{n}^{X} A_{n} P_{n}^{(1 ; 1)}(n) \frac{e^{i ! n} t}{2} \frac{1}{0(x)} \frac{u_{0}(x)}{h !_{n}} \text {, } \\
& +\frac{e^{i !_{n} t}}{2} \frac{1}{o(x)}+\frac{u_{0}(x)}{h ! n}
\end{aligned}
$$

This means that the equivalent $u_{n}$ and $v_{n}$ functions which would turn up in a quasiparticle expansion of the field operator,

$$
\begin{aligned}
& (x ; t)=\frac{a_{0}}{\overline{n_{c}}} p \frac{(x)}{o(x}{ }_{n} e^{i ! n} t_{u_{n}}(x)+{ }_{n}^{y} e^{i ! n} t_{v_{n}}(x) \\
& \mathrm{P}_{\overline{\mathrm{n}_{\mathrm{c}}}}^{\mathrm{a}_{0}} \mathrm{n}_{\mathrm{p}} \frac{\mathrm{n}(\mathrm{x})}{\mathrm{n}^{\mathrm{o}}}{ }^{\mathrm{o}}
\end{aligned}
$$

are given by

$$
\begin{aligned}
& u_{n}(x)=\frac{A_{n}}{2} \frac{1}{o(x)}+\frac{u_{0}(x)}{h !_{n}} P_{n}^{(1 ; 1)}(h) \\
& v_{n}(x)=\frac{p}{2} \frac{A_{n}}{o(x)} \frac{1}{2} \frac{u_{0}(x)}{h !_{n}} P_{n}^{(1 ; 1)}(h) ;
\end{aligned}
$$

where $A_{n}$ are normalization constants to be determined.

3.1.4. Amplitudes orthogonal to the condensate The correct eigenfunctions whose quantized amplitudes represent quasiparticles are orthogonal to the condensate wavefunction. To check this, we evaluate

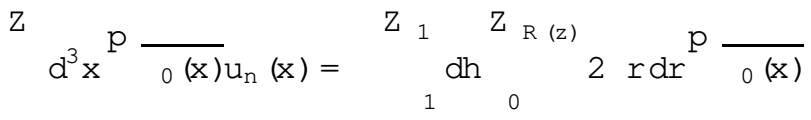

$$
\begin{aligned}
& \frac{\mathrm{A}_{\mathrm{n}}}{2} \frac{1}{\mathrm{o}(\mathrm{x})}+\frac{\mathrm{u}_{0}(\mathrm{x})}{\mathrm{h} !_{\mathrm{n}}} \mathrm{P}_{\mathrm{n}}^{(1 ; 1)}(\mathrm{h}) \\
& =\frac{A_{n}}{2}{ }_{1}^{Z_{1}} d h{ }_{0}^{Z_{R}(z)} 2 \operatorname{rdr} \frac{1}{2}+\frac{u_{0}(x)}{h ! n} P_{n}^{(1 ; 1)}(h) \text { : }
\end{aligned}
$$

We now use the Thomas-Fermi form (21) for the stationary condensate density so that

$$
\begin{aligned}
& Z_{R(z)}^{0} 2 r d r=R(z)^{2}=\frac{2\left(1 h^{2}\right)}{m !_{r}^{2}} ; \\
& Z^{0}{ }_{R(z)} \operatorname{rdru} O(x)=\frac{2}{m !_{r}^{2}}\left(1 \quad h^{2}\right)^{2}:
\end{aligned}
$$

so that, using the orthogonality property (26), equation (37) becomes

$$
\begin{aligned}
& \left.\frac{A_{n}}{2 m ! !_{r}^{2}}{ }_{1}^{Z_{1}} \text { dh }\left(1 \quad h^{2}\right) P_{n}^{(1 ; 1)}(h) \quad 1+\frac{\bar{h} !_{n}}{(1} h^{2}\right) \\
& =\mathrm{n} ; 2 \frac{{ }^{2} \mathrm{~A}_{\mathrm{n}}}{2 \mathrm{~m} !{ }_{\mathrm{r}}^{2} \mathrm{~h} ! \mathrm{n}}{ }_{1}^{\mathrm{Z}_{1}} \text { dh }\left(1 \mathrm{~h}^{2}\right)^{2} \mathrm{P}_{2}^{(1 ; 1)}(\mathrm{h}) \text { : }
\end{aligned}
$$


The nonorthogonal part-which turns up only in the quadrupole mode-represents an unobservable time dependent phase of the condensate, and is correctly treated by simply subtracting the component parallel to the condensate [9. 8], equivalent to making the replacement in the second part of the large bracket in (34) and (35) $\mathrm{P}_{n}^{(1 ; 1)}(\mathrm{h}) ! \mathrm{P}_{\mathrm{n}}^{(1 ; 1)}(\mathrm{h})$, where

$$
\begin{aligned}
& \mathrm{P}_{\mathrm{n}}^{(1 ; 1)}(\mathrm{h})=\mathrm{P}_{\mathrm{r}} \mathrm{P}^{(1 ; 1)}(\mathrm{h}) \quad \mathrm{n} \notin 2 ; \\
& \mathrm{P}_{2}^{(1 ; 1)}(\mathrm{h})=\frac{15}{56} 7 \mathrm{~h}^{2} \quad 1 ;
\end{aligned}
$$

where the norm of $\mathrm{P}_{2}^{(1 ; 1)}$ has been fixed to be the same as that of $\mathrm{P}_{2}^{(1 ; 1)}$, namely $6=7$.

It is also straightforward to check that all the $\mathrm{P}_{\mathrm{n}}^{(1 ; 1)}$ for $\mathrm{n} \quad 1$ are orthogonal to each other, so that

$$
\begin{aligned}
& \text { Z } \\
& d^{3} x\left[u_{n}(x) u_{m}(x) \quad v_{n}(x) v_{m}(x)\right] \\
& =\mathrm{Z}_{1} \mathrm{dh} \frac{2\left(1 \mathrm{~h}^{2}\right)}{\mathrm{m} !_{\mathrm{r}}^{2}} \frac{\mathrm{uA}_{\mathrm{n}}^{2}}{2 \mathrm{~h} !_{\mathrm{n}}} \mathrm{P}_{\mathrm{m}}^{(1 ; 1)}(\mathrm{h}) \mathrm{P}_{\mathrm{n}}^{(1 ; 1)}(\mathrm{h}) \\
& =\overline{m ! 2} \frac{u A_{n}^{2}}{h !_{n}}{ }_{n}, m R_{n}
\end{aligned}
$$

where

$$
R_{n}=\frac{8(n+1)}{(2 n+3)(n+2)}+n ; 2 \frac{24}{35} \frac{6}{7}^{\#)}:
$$

Since the normalization is given by

$$
\mathrm{Z}
$$$$
d^{3} x\left[u_{n}(x) u_{n}(x) \quad v_{n}(x) v_{n}(x)\right]=1
$$

we must choose

$$
A_{n}=\frac{S \overline{h ! n} m !_{r}^{2}}{R_{n} u}:
$$

3.1.5. The number of particles per quasiparticle Neglecting the zero point contribution, negligible in comparison to the thermal contribution in this case, the number of particles per quasiparticle for the mode $\mathrm{n}$ is given by

$$
\begin{aligned}
Q_{n} & =d^{3} x\left[u_{n}(x)^{2}+v_{n}(x)^{2}\right] \\
& =A_{n}^{2} \quad d^{3} x \frac{P_{n}^{(1 ; 1)}(h)^{2}}{8 o(x)}+\frac{u^{2} o(x) P_{n}^{(1 ; 1)}(n)^{2}}{2 h^{2} !_{n}^{2}}:
\end{aligned}
$$

The first term involves a divergent integral, arising from the form (21) for $o(x)$. This problem is a reflection of the failure of the linearized expansion (30) This can be fixed by noting that the true density does not go to zero at $h=1$, but reaches a value dependent on the healing length. The result is only logarithmically divergent, and any estimate of it is very much smaller than the second term, which does not diverge. We will therefore neglect this first term. 

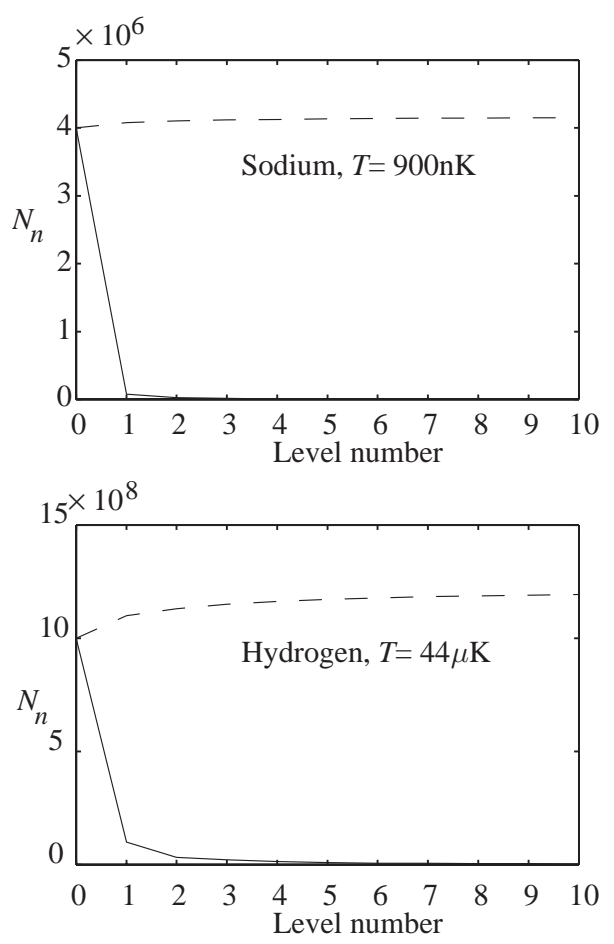

Figure 1. Comparison of atom numbers in the lowest quasiparticle modes for a sodium and a hydrogen condensate. a) Solid line: occupation of each mode; b) Dotted line: cumulative number up to the given level.

$Z_{1}$

Using the expression (21) for $0(x)$, we need the results

$\left.\operatorname{dh}\left(1 \quad h^{2}\right)^{2} \mathbb{P}_{\mathrm{n}}^{(1 ; 1)}(\mathrm{h})\right]^{2} \quad \mathrm{~S}_{\mathrm{n}}$

1

$=\frac{8(n+1)}{(2 n+3)(n+2)} \quad \frac{8(n+1)}{(2 n+3)^{2}} \quad \frac{(n+1)(n+3)}{(n+2)(2 n+5)}+\frac{n}{(2 n+1)} \quad ; \quad(n \notin 2)$

$=\frac{8}{21}$;

$(n=2)$

and using the explicit form for $A_{n}(46)$, we find

$$
Q_{n}=\frac{u S_{n}}{2 h{ }_{n} R_{n}}:
$$

Here $=\quad=u$ is the peak condensate density, which in the experiment of Fried et al. [3] was reported to be $4: 8 \quad 10^{21} \mathrm{~m}^{3}$.

\subsection{Numbers and densities for the experimental situation}

Wsing the result (25) and the experimental value for $!_{z}$, we have $!_{n}=2 \quad 10 \cdot 2 \mathrm{~Hz}$ $\overline{n(n+3)=4}$, leading to $Q_{n} \quad 3776 S_{n}=\overline{n(n+3)} R_{n}$. This would lead to

$$
N_{n}=Q_{n} \frac{k T}{h ! n} \quad \frac{6: 9 \quad 10^{8} S_{n}}{n(n+3) R_{n}}
$$



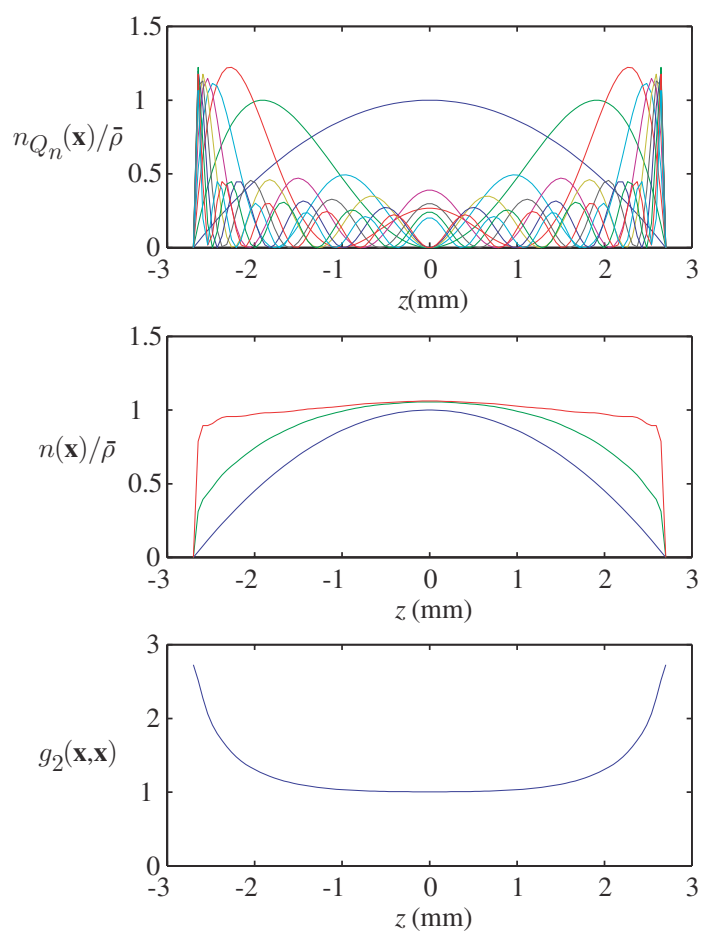

Figure 2. Plots showing: a) The individual contributions to the total density from the condensate and the first 10 eigenfunctions; b) From bottom to top, the condensate density, the total density, and the total density multiplied by $g_{2}(x ; x)$ (Normalization for both chosen so that the peak density arising from the condensate wavefunction is 1$)$; c) The $g_{2}(x ; x)$ arising from all of the components.

particles in each mode. In Fig.11 we illustrate the contributions to the particle numbers from the condensate and the first two condensate modes for the hydrogen condensate and for a typical MIT sodium condensate [7], whose geometry is a much less extreme cigar shape. The net contribution from the quasiparticle modes is about $2 \%$ of the number in the condensate mode for sodium, but for Hydrogen approaches $20 \%$ of the number in the condensate mode.

Notice that convergence appears after about 10 modes.

3.2.1. Particle density arising from quasiparticles The contribution to $n(h)$ arising from the quasiparticles is simply of the form of the integrand in (47) for each mode, with the resulting overall density taking the form

$$
n_{Q}(x)={ }_{n}^{x} \frac{A_{n}^{2} k T}{h ! n} \frac{\left(P_{n}^{(1 ; 1)}(h)^{2}\right.}{80(x)}+\frac{u_{0}^{2}(x) P_{n}^{(1 ; 1)}(h)^{2}}{2 h^{2} !_{n}^{2}}
$$

We will need the anomalous average as well, that is the term

$$
\left.\mathrm{m}_{Q}(\mathrm{x})\right)^{\mathrm{D}} \sim(\mathrm{x})^{2^{\mathrm{E}}}
$$

and this is quite readily shown to be

$$
m_{Q}(x)={ }_{n}^{x} \frac{A_{n}^{2} k T}{h ! n} \frac{\left(P_{n}^{(1 ; 1)}(h)^{2}\right.}{80(x)} \quad \frac{u^{2} o(x) P_{n}^{(1 ; 1)}(h)^{2}}{2 h^{2} !_{n}^{2}}
$$



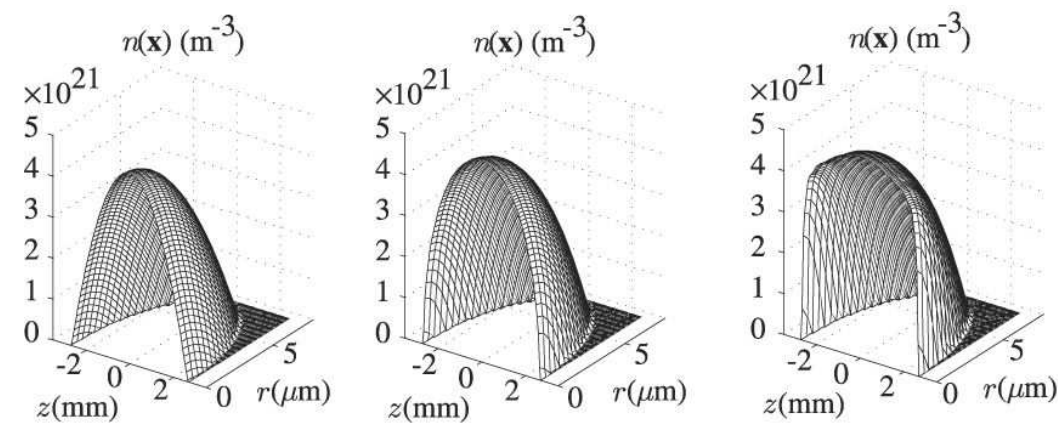

Figure 3. Plots vs. $r$ and $z$ showing: a) The condensate density (in the Thomas-Fermi approximation); b) The density arising from the condensate plus first 10 quasiparticle states (thermally occupied); c) The effective density, obtained from b) by multiplying by $g_{2}(x ; x)$

In the degree of approximation we are using, in which the first terms in the bracketed parts of the right hand sides of (54) and (56) are neglected, it follows that $\mathrm{m}_{Q}(\mathrm{x})=\mathrm{n}_{Q}(\mathrm{x})$-the anomalous term is thus very important.

3.2.2. Computation of $g_{2}(x ; x)$ The quasiparticle contribution to the field operator

$$
\sim(x)={ }_{n}^{x} \quad n e^{i ! n t} u_{n}(x)+{ }_{n}^{y} e^{i ! n} v_{n}(x)
$$

is composed of operators $n ; \quad \frac{\mathrm{y}}{n}$, for which the density operator is quantum Gaussian with zero mean, so the Gaussian factorization of the the four point correlation function is

$$
\begin{aligned}
\mathrm{D} \sim \mathrm{y}(\mathrm{x}) \sim \mathrm{y}(\mathrm{x}) \sim(\mathrm{x}) \sim(\mathrm{x}) \stackrel{\mathrm{E}}{=} & 2 \mathrm{n}_{Q}(\mathrm{x})^{2}+\min \mathrm{Q}(\mathrm{x})^{2} j \\
& 3 \mathrm{n}_{Q}(\mathrm{x})^{2}:
\end{aligned}
$$

Thus, for the full field operator

$$
\begin{aligned}
& { }^{y}(x){ }^{y}(x)(x)(x)=0(x)^{2}+40(x) n_{Q}(x) \\
& +20(x) m_{Q}(x)+2 n_{Q}(x)^{2}+\dot{m} n_{Q}(x)^{2} j \\
& o(x)^{2}+2 \circ(x) n_{Q}(x)+3 n_{Q}(x)^{2}
\end{aligned}
$$

Noting now that the particle density is

$$
\begin{aligned}
n(x)= & 0(x)+n_{Q}(x) \\
& 0(x)[1+q(x)]
\end{aligned}
$$

which defines $q(x)$, we see that

$$
g_{2}(x ; x)=1+2 \frac{q(x)}{1+q(x)}^{2}:
$$

Notice that the possible values of $g_{2}(x ; x)$ are between 1 and 3 , and that $g_{2}(x ; x)$ does depend on position.

Using these expressions we can now compute both $n(x)$ and $g_{2}(x ; x)$, and these are illustrated in Fig.2. 


\subsection{Interpretation}

Fig. 3 consists of three plots, to illustrate the two major effects arising from this calculation. Comparing a) with b), it can be seen how the condensate density is modified both in shape and size by the excitation of the quasiparticles. Comparison of b) with c) shows how inclusion of the factor of $g_{2}(x ; x)$ would modify the signal from the experiment not by simply multiplying by a factor of 2 as has been assumed in the experiment.

\section{Conclusion}

The results of this calculation are only of indicative interest for the following reasons:

a) The occupations of the quasiparticle levels are large enough that the simple Bogoliubov formulation may need modification.

b) There is as yet no justification for simply including the factor of $g_{2}(x ; x)$ as the appropriate correction for correlations.

c) The $g_{2}(x ; x)$ computed is the result of an effective interpolation (given by (64)) of $g_{2}(x ; x) \quad 3$ for the quasiparticles and $g_{2}(x ; x) \quad 1$ for the condensate. It can be shown in fact that the experimental frequency shift which results in the hydrogen condensate experiments is different for condensate and quasiparticles, so that there is no simple interpretation in terms of a single frequency shift determined by $g_{2}(x ; x)$.

These matters are be attended to in our second paper 㺻, but it is already clear that the correct interpretation of the cold-collision shift in these experiments is a matter of great significance.

\section{Acknowledgments}

We wish to thank Dan Kleppner, Tom Greytak, Stephen Moss, Lorenz Willman and Kendra Vant for useful discussions about the hydrogen condensate experiments and hospitality in MIT. This research was supported by the Royal Society of New Zealand under the Marsden Fund Contract PVT-902.

\section{References}

[1] Fliesser, M., Csordás, A., Szépfalusy, P., and Graham, R. Phys. Rev. A 56 (1997), R2533.

[2] Fried, D. G. Bose-Einstein Condensation of Atomic Hydrogen. PhD thesis, Massachusetts Institute of Technology, 1999.

[3] Fried, D. G., Killian, T. C., Willmann, L., Landhuis, D., Moss, S. C., Kleppner, D., and Greytak, T. J. Phys. Rev. Lett. 81, 18 (1998), 3811.

[4] Gardiner, C. W., and Bradley, A. S. Theory of the cold collision frequency shift in $1 S-2 S$ spectroscopy of Bose-Einstein-condensed and non-condensed hydrogen. cond-mat/0108451, 2001.

[5] Killian, T. C. 1s-2s Spectroscopy of Trapped Hydrogen: The Cold Collision Frequency Shift and Studies of BEC. PhD thesis, Massachusetts Institute of Technology, 1999.

[6] Killian, T. C., Fried, D. G., Willman, L., Moss, D. L. S. C., Greytak, T. J., and Kleppner, D. Phys. Rev. Lett. 81, 18 (1998), 3807.

[7] Miesner, H.-J., Stamper-Kurn, D. M., Andrews, M. R., Durfee, D. S., Inouye, S., and Ketterle, W. Science 279 (1998), 1005.

[8] Morgan, S. A. A Gapless Theory of Bose-Einstein Condensation in Dilute Gases at Finite Temperature. PhD thesis, University of Oxford, 1999.

[9] Morgan, S. A., Choi, S., and Burnett, K. Phys. Rev. A 57 (1998), 3818.

[10] Zaremba, E. Phys. Rev. A 57 (1998), 518. 

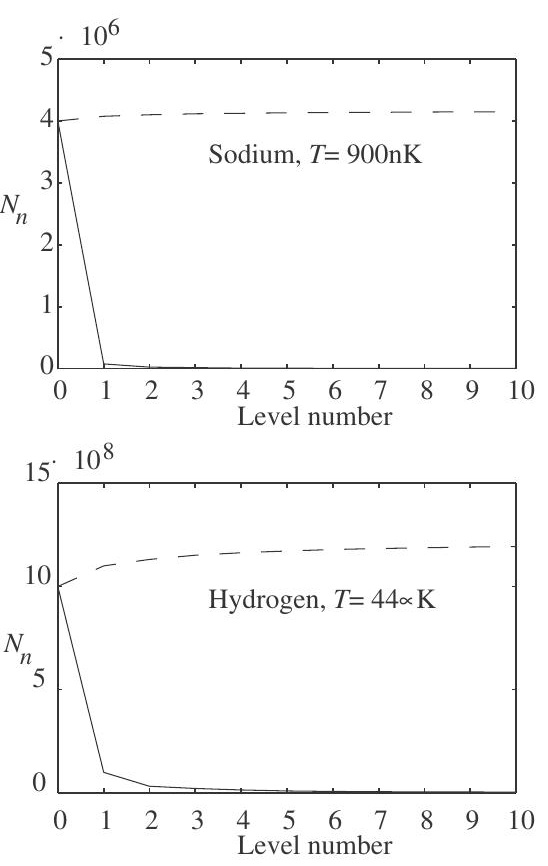